\title{
Subject Index Vol. 209, 1995
}

Acute angle-closure glaucoma 220

- $\quad$ idiopathic blind-spot syndrome 217Age-dependent trabecular pigmentation

190 Age-related macular degeneration 194, 302 Aging 199 AIDS 260

Angle-closure glaucoma 223 Animal eye 336 Anophthalmic socket 319 Anterior chamber aqueous 56

optic neuropathy 346

uveitis 53

Antineutrophil cytoplasmic antibody 172

Antiproliferative drugs 212

Aqueous flare 145

Argon laser 21

Aspergiïlus flavus 109

Astigmatism 136

Atopic dermatitis 160

Autogenic relaxation 122

B-cell lymphoma 331 Bacterial adhesion 315 Behçet’s disease 270 Biomaterials in ocular surgery 315 Biopsy 331 Blind spot 217 Blood flow 233

pressure 88 Blood-aqueous barrier 145 Blood-retinal barrier 145

- stabilisation 149 Bovine factor VIII derivative 149

- VHI-derived peptide fraction 117

Calcium 14

antagonist(s) 267, 336

channel blockers 11 Canalicular reconstruction 27 Capsular glaucoma 21 Cataract surgery 270

Cavernous sinus 292

Central retinal artery occlusion 37

- $\quad$ - vein occlusion 106,248Chamber angle pigmentation 21Childbirth 37Chloroquine 79Cholesterol 60

Choroid 284

Choroidal detachment 220

- $\quad$ melanoma 7Ciliary epithelial tear 160

Coats' disease 44 Colour Doppler imaging 117 Complicated cataract 270 Computed tomography 182, 228 Cone electroretinography 251 Congenital nystagmus 1 Conjunctiva 47 Contrast sensitivity 194 Cornea 47

Cornea-marking device 25 Corneal degeneration, band-shaped spheroidal 96 dystrophy, gelatinous drop-like 96

endothelium 17

ulcer 155 Corticosteroids 40 Cushing's disease 88 Cytomegalovirus 40, 260

Dacryocystography 228 Darier-White disease 47 Dexfenfluramine 223 Diabetes mellitus 53

Diabetic retinopathy 56, 145 Diffuse clinically significant diabetic 
macular edema 64 Diltiazem 336 Disposable electrode 299 Double running suture 25 Down gaze palsy 225 Drusen 194 Dyschromatopsias, inborn 242

Elderly patients 203 Electron microscopy 44, 336 Electroretinogram 101,187,299

Electroretinography 79, 92 Empty sella syndrome 106 Endogenous endophthalmitis 109

- $\quad$ fluorescence 7Endophthalmitis 165Episcleritis 256Excimer laser 17Exfoliation syndrome 199Exophthalmos 172Exposure of implant 319Extraocular muscle(s) 1,336Exudative retinal detachment 220Eyelid 47

Fibroblasts 212

Fluorescein angiography 88,92, 101, 145, 217

Foam cell 44

Fuchs' heterochromic iridocyclitis 289

Ganglion cells 233 Gd-DTPA 292 Genetic eye disease 278 Glaucoma 11,165,177,190 Glycogen storage disease 92 Gonioscopy 132 Graves orbitopathy 182 Group training 122 Grouped pigmentation 101

Hemangioma, choroidal 284 Hemodialysis 14 Hemorrhage, subconjunctival 289 Herpes zoster 267 High-density lipoprotein 60 High-tension glaucoma patients 129 Histopathology 331 Human eye 331 Hydroxyapatite implant 319 Hypertensive retinopathy 187

Immunization, passive 340 Immunoglobulin subclass deficiency 40 Immunology 53 Inheritable glaucoma 68 Intraocular pressure 70, 199,233,275

- $\quad$ tumor 331Iodine uptake 84Iontophoresis 84

Iridocorneal angle pigmentation 190

Ischemia 248

I shihara plates 242

Juvenile open-angle glaucoma 68

Keratitis, herpetic 340

- $\quad$ Solaris 329Keratoconus 278Keratosis follicularis 47

Lacrimal sac diverticulum 228

- $\quad$ stents 27

Laser flare cell meter 56,145 Lateral rectus muscle 336 Lens, bacterial adhesion 315

Leukoemboli, complement-induced 37 Lipidosis 79

Liquid perfluorocarbons 306 Local anesthesia 208 Low-density lipoprotein 60

Macroaneurysms 248 Macular edema 60

- $\quad$ hole 203Magnesium 11

Magnetic resonance imaging 182, 225, 228,

292 Masquerade syndrome 331 Measurement apparatus 113 Meibomian carcinoma 280

Modified grid laser 64 Multifocal choroiditis 217 Multiple evanescent white-dot syndrome

217

- $\quad$ traction sutures 27Myopia 17

Neovascular glaucoma 141

- $\quad$ macular degeneration 194Nicardipine 267Non-Hodgkin's lymphoma 331Non-

proliferative diabetic retinopathy 149Normal-tension glaucoma patients 129

Ocular calcification 14

circulation 70

infections 315

movement 208

trauma 165 Ofloxacin 155 Open-angle glaucoma 122 Optic disc measurement 177 
nerve 233

neuritis 260

Oral mucous membrane 27 Orbital pseudotumor 172 Oscillatory potentials 187 Outpatient

clinical setting 113

Pancryopexy 141 Parathyroid hormone 14 Pathological lesions 306 Pattern electroretinogram

233, 299 Penetrating keratoplasty 25 Perfluorodecalin 306

Periaqueductal lesion 225 Periodontitis 109 Pharmacological therapy 149 Phosphate 14

Photocoagulation treatment 60 Photorefractive keratectomy 17 Phytanic acid storage disease 251

Pituitary adenoma 88 Porcine eye model 84 Postherpetic neuralgia 267 Postural change 129

Primary angle closure glaucoma 113 Proliferative vitreoretinopathy 44,212 Prolonged

tamponade 306 Protein concentration 56 Pseudomonas aeruginosa 155 Pseudo-ocular

histoplasmosis syndrome

217 Pseudophakia 165 Psychophysical functions 302 Pulseless disease 346 Pupil-blocking force 113 Purtscher's retinopathy 37

Rabbit(s) 212,275,306

Radial keratotomy 165

Radiation therapy 280

Radiotherapy 331

Rats 79

Refsum's disease 251

Retina 101,306

Retinal detachment 160,323

dystrophy 278

pigment epithelium 7, 88

venous stasis 106 Retinitis 40,260

pigmentosa 47 Retinoblastoma 284 Retinopathy 88

Rhegmatogenous retinal detachment 203 Rheology 70

Risk index 113

Rod electroretinography 251

Rubeosis iridis 141

Scleral buckling surgery 323

- $\quad$ - technique 160 Scleritis 256

Sebaceous gland carcinoma 280

Serotonin 223

Serum lipid fractions 60

Simple glaucoma 21

Spontaneous carotid cavernous fistula 220

Stereovideograph 177

Steroid therapy 182

Strabismus 208, 336

- $\quad$ surgery 136Sub-Tenon approach 208Suction cup method 70,233Sun beds 329

Suramin 212

T-lymphocyte subsets 53 Takayasu disease 346 Tear meniscus height 27 Tonometry 275 Tono-

Pen 275 Trabeculectomy 132 Trabeculoplasty 21 Tumors 88

Ultrasonography 228 Ultrastructural study 1 Ultraviolet exposure 329 Uveitis 270

Vasculitis 172 Vasospasm 11 VECP 233 
Venous thrombosis 292 Visaline ${ }^{\circledR} 302$ Visual acuity 323

evoked cortical potentials 70, 101

field(s) 11,129

imagination 122 Vitrectomy 165

Wegener's granulomatosis 172

3. 352

Subject Index Vol. 209

1995 